\title{
Modelling urban traffic dynamics based upon the variational formulation of kinematic waves
}

\author{
Andy H.F. Chow* \\ Shuai Li \\ Centre for Transport Studies, University College London \\ London WC1E 6BT, United Kingdom \\ W.Y. Szeto \\ Department of Civil and Environmental Engineering, The University of Hong Kong \\ Pok Fu Lam, Hong Kong \\ David Z.W. Wang \\ School of Civil and Environmental Engineering, Nanyang Technological University \\ Nanyang Avenue, Singapore
}

December 19, 2014

\begin{abstract}
This paper presents a dynamic traffic modelling framework based upon the variational formulation of kinematic waves. We compare the effectiveness of this relatively recent numerical method with the traditional Godunov-based cell transmission method on various aspects including modelling shocks, dispersion of vehicle platoons, moving bottlenecks, and traffic characteristics with respect to real-world observations made in Central London, UK. The results suggest that the variational method is able to produce high quality estimates both theoretically and empirically. This study opens up a new research direction in the area of urban traffic modelling and optimisation.
\end{abstract}

Keywords: Godunov scheme, Cell transmission method (CTM), variational method, urban journey times, multi-modal networks

\footnotetext{
*andy.chow@ucl.ac.uk
} 


\section{Introduction}

Understanding the characteristics of urban congestion is a prerequisite for deriving effective transport policies and management plans. A previous study reveals that $25 \%-30 \%$ of congestion observed in Central London (UK) could be reduced by effective traffic management (Chow et al., 2014). Compared with motorways, there has not been much research conducted on urban streets due to the complexity of urban traffic dynamics and lack of relevant data. Recently, the increasing availability of data from different sources has enabled us to carry out more comprehensive research on urban network traffic modelling. Among all traffic models proposed in the literature, kinematic wave model remains the most widely accepted one due to its ability to capture realistic traffic behaviour such as spillover and propagation of shockwaves with parsimonious mathematical structure. The kinematic wave model is proposed by Lighthill and Whitham (1955) and Richards (1956), and hence it is also known as the LWR (Lighthill-Whitham-Richards) model. In the LWR model, traffic flow $f(x, t)$ at location $x$ and time $t$ is related to the associated density value $\rho(x, t)$ through a flow-density mapping $\Phi$ which is known as the fundamental diagram:

$$
f(x, t)=\Phi[\rho(x, t)] .
$$

The evolution of density is governed by the following conservation law (LeVeque, 1992):

$$
\frac{\partial \rho(x, t)}{\partial t}+\frac{\partial f(x, t)}{\partial x}=0 .
$$

The partial differential equation (PDE) depicted in Equation (2) is usually solved by some first-order Godunov (1959) discretization schemes which include the popular cell transmission method (CTM) (Daganzo, 1994). Since Daganzo (1994), CTM has been applied in a number of studies ranging from freeways (e.g. Gomes and Horowitz (2006), Chow et al. (2008)) to urban networks (e.g. Ziliaskopoulos (2000), Lo and Szeto (2002), Chow et al. (2010)). Despite its popularity, CTM could produce error at discontinuity (i.e. shock) in its solution (Daganzo (2006), Mazare et al. (2011)). Moreover, it is also known that CTM is ineffective in modelling moving bottlenecks induced by slow-moving vehicles such as trucks and buses in the urban area (Lebacque et al. (1998), Mazare et al. (2011)).

Daganzo (2005a) and Daganzo (2005b) propose an alternative solution method to LWR model which is known as the variational method. The variational method integrates Equations (1) and (2) into one single Hamilton-Jacobi-Bellman (HJB) equation (see Equation (15)). Following this, the LWR model can be solved by dynamic programming (see Equation (18)) with any concave fundamental diagram $\Phi$ under this variational formulation. The variational method shows significant improvement in terms of solution 
accuracy with respect to the analytical solution over the Godunov scheme (Daganzo and Menendez, 2005). Daganzo and Menendez (2005) and later Mazare et al. (2011) also present an analysis of moving bottleneck on freeways using the variational method that is not easy to capture on a Godunov platform (see Lebacque et al. (1998)). Daganzo (2006) and Laval and Leclercq (2013) discuss the duality of the variational formulation in which the solution of the HJB can be expressed in Lagrangian and 'time' ('T-model') coordinates in addition to the traditional Eulerian coordinate. Mehran et al. (2012) present a data fusion algorithm based upon the variational formulation of kinematic waves with triangular fundamental diagram.

Most previous studies on the variational formation of kinematic waves are either theoretical analysis or applications on freeways, there have not been many studies on urban street networks. This study contributes to the literature by presenting a variationalbased network modelling framework which considers various aspects in urban area including traffic signals, slow-moving buses, and dispersion of platoons. We also compare the performance of this variational method with the established CTM over a range of scenarios and a case study in Central London, UK. This paper is organised as follows: Section 2 presents a modelling framework for dynamic network traffic including a discussion on CTM and variational method. Section 3 presents a range of numerical experiments that aim to compare the characteristics of CTM and the variational method over different applications. Section 4 presents the case study with traffic data collected from Tottenham Court Road in Central London, UK. Finally, Section 5 presents some concluding remarks.

\section{Dynamic network model}

A dynamic network model consists of a node component and a link component as shown in the following sections.

\section{$2.1 \quad$ Node model}

Consider a specific node $m$ with $I_{m}$ incoming links and $J_{m}$ outgoing links. Following Nie et al. (2008), Chow et al. (2010), and others, we define a split matrix $\boldsymbol{\beta}_{k}=\left[\beta_{i j}(k)\right]$, which has a dimension of $\left(I_{m} \times J_{m}\right)$, for the node. The split ratio is updated regularly (say, every 15-min) over a sampling period $k$ based on field observations. The element $\beta_{i j}(k)$ in the split matrix $\boldsymbol{\beta}_{k}$ specifies the proportion of traffic on each incoming link $i$, where $i=1,2, \ldots, I_{m}$, that is heading to each outgoing link $j$, where $j=1,2, \ldots, J_{m}$, through node $m$ during time interval $k$. The principle of conservation requires that

$$
\sum_{j=1}^{J_{m}} \beta_{i j}(k)=1
$$


for all $i=1,2, \ldots, I_{m}$. The split ratios $\boldsymbol{\beta}_{k}$ may also be determined endogenously by traffic conditions in the network through a dynamic traffic assignment model (see Lo and Szeto (2002), Heydecker and Addison (2005), Chow (2009)). However, detailed discussion of dynamic traffic assignment model is beyond the scope of the present paper. Without consideration of excessive blocking (i.e. traffic can be freely flowing through node without restraint due to downstream queue), the traffic volume $f_{i j}(k)$ during time $k$ flowing from incoming link $i$ to outgoing link $j$ is determined as:

$$
f_{i j}(k)=\beta_{i j}(k) \lambda_{i}(k)
$$

where $\lambda_{i}(k)$ is total traffic volume on link $i$ wanting to get through node $m$ during time $k$.

If the node is controlled by a traffic signal, the signalling effect can be captured by associating a binary variable $\gamma_{i j}(t)$ with the term on the right-hand-side in Equation (4) (Chow et al., 2010), i.e.

$$
f_{i j}(k)=\beta_{i j}(k) \lambda_{i}(k) \gamma_{i j}(t)
$$

in which:

$$
\gamma_{i j}(t)= \begin{cases}1, & \text { if movement from } \mathrm{i} \text { to } \mathrm{j} \text { is given a green signal } \\ 0, & \text { if movement from } \mathrm{i} \text { to } \mathrm{j} \text { is given a red signal }\end{cases}
$$

The formulation (6) works for both fixed-time plans (which operate according to a predefined timing plan) and responsive controllers which operate based on real-time vehicle actuations (Chow et al., 2010). Moreover, the corresponding total outflow $\mu_{j}(k)$ through node $m$ to link $j$ will be

$$
\mu_{j}(k)=\sum_{i=1}^{I_{m}} f_{i j}(k)
$$

The formulations (4) and (7) can be extended to capture excessive blockage through considering the available space at the downstream (Nie et al. (2008), Kurzhanskiy et al. (2009), Zhang et al. (2013)). Let $Q_{j}(k)$ be the maximum flow that can be accommodated by link $j$ at time $k$. This $Q_{j}(k)$ can be estimated by various link traffic models (see Section 2.2) while for now we assume that it is known. Nie et al. (2008) and Kurzhanskiy et al. (2009) propose to adjust the flow $f_{i j}(k)$ in Equation (4) as

$$
\hat{f}_{i j}(k)=\frac{\min \left(\mu_{j}(k), Q_{j}(k)\right)}{\mu_{j}(k)} f_{i j}(k),
$$


It is noted that if $\mu_{j}(k) \leq Q_{j}(k)$, which implies there is enough capacity on link $j$ to accommodate the incoming traffic, then Equation (8) will produce an identical estimate of $f_{i j}(k)$ as Equation (4). If $\mu_{j}(k)>Q_{j}(k)$ (i.e. excessive blockage occurs), Equation (8) states that contributions $f_{i j}(k)$ from all links $i$ should be reduced by a common factor $\frac{Q_{j}(k)}{\mu_{j}(k)}$. This guarantees the predefined split ratio matrix $\boldsymbol{\beta}$ remains unchanged. Following the adjustment by Equation (8), we also need to adjust $\lambda_{i}(k)$ accordingly as

$$
\hat{\lambda}_{i}(k)=\sum_{j=1}^{J_{m}} \hat{f}_{i j}(k),
$$

where $\hat{\lambda}_{i}(k) \leq \lambda_{i}(k)$

\subsection{Link model}

The link model describes the dynamic propagation of traffic along the link. The point queue model (Zhang et al., 2013), which is also known as the bottleneck model (Vickrey, 1969) or deterministic queueing model (Chow, 2009), is one of the simplest representations of link traffic propagation. The point queue model considers each link to be consisting of two parts: a freely flowing part with a flow-invariant travel time combined with a queue of traffic at its downstream end being discharged with a maximum rate. The traffic queue is considered to be stacking up vertically and hence takes no physical space of the road. Recent studies (Han et al. (2013a), Han et al. (2013b)) show that this point-queue model can be implemented under the variational method that we are going to discuss in the later sections. Nevertheless, ignoring the spatial effect of traffic can lead to ill-representation of physical behaviour as shown by a number of previous studies including Szeto and Lo (2006) and Zhang et al. (2013). Considering plausibility, this study will use the LWR model and the following sections will present two of the solution schemes: cell transmission and variational methods.

\subsubsection{Cell transmission method}

Cell transmission method (CTM) is proposed by Daganzo (1994) and Daganzo (1995) as a Godunov based discretization scheme of the LWR model. Under CTM, the entire road network is discretized into a collection of sections or 'cells' as shown in Figure 1 in which the cells are numbered from downstream 0 , to upstream $N-1$. Given the outflow $f_{i}(t)$ from each cell $i$ in time interval $t$, we can derive the state equation on the corresponding density $\rho_{i}$ from conservation law as 


$$
\rho_{i}(t+1)=\rho_{i}(t)+\frac{\Delta t}{\Delta x_{i}}\left[f_{i+1}(t)-f_{i}(t)\right]
$$

where $\Delta t$ and $\Delta x_{i}$ are respectively the lengths of the simulation time step and cell $i$. Equation (10) can indeed be regarded as a discrete version of Equation (2). The flow $f_{i}(t)$ between each pair of cells $(i, i-1)$ is related to the associated densities $\left(\rho_{i}, \rho_{i-1}\right)$ through a triangular fundamental diagram (Figure 2) at each cell. Each cell $i$ is associated with a capacity $Q_{i}$ which specifies the maximum flow that can be discharged from that cell, and a jam density $\bar{\rho}_{i}$ which is the maximum traffic density that can be stored in the cell. When there is no congestion, traffic moves through cell $i$ at a free flow speed, $v_{i}$. The quantity $w_{i}$ specifies the backward propagation speed of congestion along cell $i$. The density associated with the capacity flow is known as the critical density $\rho_{c i}$.

With this piecewise linear specification, the outflow $f_{i}(t)$ from cell $i$ is determined from the traffic densities $\rho_{i}$ and $\rho_{i-1}$ as

$$
f_{i}(t)=\min \left\{v \rho_{i}(t), Q_{i}, Q_{i-1}, w\left[\bar{\rho}_{i-1}-\rho_{i-1}(t)\right]\right\},
$$

which can be viewed as a mathematical expression of the triangular flow-density relationship depicted in Figure 2. The outflow function (11) is due to the assumption of the triangular fundamental diagram shown in Figure 2. As shown in Laval (2004), Szeto (2008), Jin et al. (2009), Jabari and Liu (2012), and others, the outflow function can be generalized as

$$
f_{i}(t)=\min \left\{S_{i}(t), R_{i-1}(t)\right\}
$$

where $S_{i}(t)$ and $R_{i-1}(t)$ are regarded as the sending and receiving functions respectively. The sending and receiving functions represent respectively the traffic demand for advancing from upstream $i$ to downstream $i-1$, and the supply at the downstream $i-1$ for receiving incoming traffic from the upstream $i$. The general form shown in (12) allows the use of different forms of fundamental diagrams in addition to the triangular ones.

The cell transmission method has been applied to a number of studies on urban traffic modelling and optimisation (see: Ziliaskopoulos (2000), Szeto and Lo (2006), Chow et al. (2010), Zhu et al. (2013)). Nevertheless, a weakness of CTM for traffic modelling is that numerical errors arise at discontinuities (i.e. 'shocks') of its solutions. The flow and density profiles are smeared in regions around the shocks and this feature is termed as 'viscosity' by LeVeque (1992). Moreover, the space-time discretization under CTM is limited by the CFL (Courant-Friedrichs-Lewy) condition (Courant et al., 1928) which requires the time step size $\Delta t$ is set such that $\Delta t \leq \min _{i} \frac{\Delta x_{i}}{v_{i}}$, where $\min _{i} \frac{\Delta x_{i}}{v_{i}}$ refers to the smallest ratio of cell length to the associated free-flow speed along the section. This is 
to ensure the numerical stability and non-negativity of traffic quantities by constraining traffic not travel further than the length of the cell in one simulation time step. It is also revealed that CTM, as a Godunov scheme, is ineffective in modelling moving bottleneck, say congestion caused by slow moving buses in urban network (Lebacque et al., 1998).

\subsubsection{Variational method}

The variational method can be dated back to the seminal work by Newell (1993) which proposes a simplified version of kinematic wave model and expresses the solutions in terms of cumulative count of traffic $N(x, t)$ at $x$ and $t$, where by definition

$$
\begin{gathered}
\left.\frac{\partial N}{\partial t}\right|_{(x, t)}=q(x, t), \\
-\left.\frac{\partial N}{\partial x}\right|_{(x, t)}=\rho(x, t) .
\end{gathered}
$$

It is shown in Newell (1993) that cumulative traffic that passes by a location at a particular time can be determined by its upstream and downstream boundary conditions by simple translations of cumulative curves. This simplified kinematic wave theory has been applied to development of efficient network loading models (Yperman (2007), Balijepallia et al. (2013)), sensitivity analysis of traffic control (Chow and Lo, 2007), and urban traffic optimisation (Han et al., 2014). Daganzo (2005a) and Daganzo (2005b) propose an numerical scheme using dynamic programming for solving LWR model based upon Newell (1993) and which is now known as the variational method. Following specifications in Equations (13) and (14), the LWR equations (1) and (2) can be integrated as:

$$
\left.\frac{\partial N}{\partial t}\right|_{(x, t)}=\Phi\left[-\left.\frac{\partial N}{\partial x}\right|_{(x, t)}\right]
$$

Equation (15) is recognised as a Hamilton-Jacobi equation where the fundamental diagram $\Phi$ is regarded as the Hamiltonian function. Given the initial and boundary conditions, it is shown that this Hamilton-Jacobi equation (15) can be solved by dynamic programming with unprecedented accuracy and efficiency if the underlying fundamental diagram is concave (Daganzo, 2005b). To solve this variational formulation, Daganzo $(2005 a)$ introduces the following cost function in wave speed $u(x, t)=\left.\frac{\partial q}{\partial \rho}\right|_{(x, t)}$ over $(x, t)$ :

$$
R(u, x, t)=\sup _{\rho}(\Phi(\rho, x, t)-u(x, t) \rho)
$$

This cost function $R$ is recognised as a Legendre-Fenchel transformation of the fundamental diagram $\Phi$ (Daganzo (2006), Mazare et al. (2011)). As explained in Daganzo 
(2005a), physically the function $R$ can be interpreted as the maximum rate at which traffic can pass an observer moving with speed $u$ at $(x, t)$. To illustrate this variational method, we now consider a link which starts at location $x_{0}$ and ends at $x_{1}$. We also define $\mathbb{B}$ be the set of initial condition $N(x, 0)$ at time $t=0$ for all locations $x$; boundary condition $N\left(x_{0}, t\right)$ at upstream end $x_{0}$; boundary condition $N\left(x_{1}, t\right)$ at downstream end $x_{1}$. Given this set of boundary values $\mathbb{B}$, the HJB Equation (15) can be solved with the cost function (16) as (Daganzo (2005b), Laval and Leclercq (2013)) :

$$
N(x, t)=\min _{\mathbb{B}}\left\{N_{\mathbb{B}}\left(x_{\mathbb{B}}, t_{\mathbb{B}}\right)+\left(t-t_{\mathbb{B}}\right) R\left(\frac{x-x_{\mathbb{B}}}{t-t_{\mathbb{B}}}\right)\right\}
$$

where $N_{\mathbb{B}}$ is the set of known values of $N$ given at $\left(x_{\mathbb{B}}, t_{\mathbb{B}}\right)$ on boundary $\mathbb{B}$. Equation (17) is known as the Lax-Hopf formula (Mazare et al., 2011), and it can be solved by a number of effective and high quality solution algorithms including the event-based gridfree algorithm proposed by Mazare et al. (2011) that does not require predefinition of a computational space-time grid. A disadvantage of the event-based algorithm by Mazare et al. (2011) is that it cannot incorporate space-time dependent fundamental diagrams and hence it is inconvenient for modelling local and temporary events such as traffic lights and incidents. Applying the principle of optimality in dynamic programming (DP), Daganzo (2005b) presents a forward DP-based method for solving Equation (17). The DP-formulation can be written for a general concave fundamental diagram over a discrete space-time grid as:

$$
N(x, t)=\min _{u \in \mathbb{U}}\{N(x-u \Delta t, t-\Delta t)+\Delta t R(u)\},
$$

where $\mathbb{U}$ is the set of all possible wave speeds in the fundamental diagram $\Phi$. Unlike Godunov schemes and CTM, the spatial interval $\Delta x$ is endogenously determined from the time step $\Delta t$ through the term ' $u \Delta t$ ' with the set of shockwave speeds $u \in \mathbb{U}$.

Consider a simple case where $\Phi$ is triangular as the one depicted in Figure 2 in which the wave speed $u=\frac{x-x_{\mathbb{B}}}{t-t_{\mathbb{B}}}$ can only take two possible values: $v$ (forward) and $w$ (backward), the cost function $R$ becomes:

$$
R(u, x, t)= \begin{cases}0, & \text { if } u=v \\ w \bar{\rho}, & \text { if } u=w\end{cases}
$$

for all $(x, t)$. Equation (18) can then be reduced to:

$$
N(x, t)=\min \{N(x-v \Delta t, t-\Delta t), N(x-w \Delta t, t-\Delta t)+\bar{\rho} w \Delta t\},
$$

which is consistent with the theory presented in Newell (1993). It is shown that, unlike 
CTM, solution derived from (20) with triangular $\Phi$ is exact.

Effect of traffic lights at a specific location $x^{*}$ or moving bottlenecks (e.g. slowing buses) can be captured in Equation (18) through introducing 'shortcuts' (Daganzo, 2005b). A traffic light at $x^{*}$ can be modelled by introducing the following revised cost function $R^{*}\left(0, x^{*}, t\right)$ associated with wave speed $u=0$ at $x^{*}$ :

$$
R^{*}\left(0, x^{*}, t\right)= \begin{cases}q_{\max }, & t \in \mathbb{G} \\ 0, & t \in \mathbb{R}\end{cases}
$$

The idea of cost function (21) is indeed the same as (6) which regulates the capacity flow at $x^{*}$ according to a predefined timing plan. To simulate the effect of a moving bottleneck $C$, which can be a slow moving bus or truck on the road, with a trajectory $x=C(t)$ over time $t$, one can introduce the following shortcut:

$$
R^{*}(u, x, t)= \begin{cases}R_{C}(t), & \text { if } x=C(t), u=\frac{d C}{d t}, \\ R(u, x, t), & \text { otherwise, }\end{cases}
$$

where $0 \leq R_{C}(t) \leq R\left(\frac{d C}{d t}\right)$, and $R_{C}(t)$ physically represents the maximum passing rate of traffic that can pass through the moving bottleneck $C$.

\section{$3 \quad$ Numerical examples}

This section presents some numerical experiments comparing traffic characteristics produced by the two numerical methods.

\subsection{Viscosity at shocks}

Figures 3 and 4 show the density maps generated respectively by CTM and variational method over time $t$ along a link which is 0.5 -mile with a traffic light located in the middle of it. For the variational method, the density maps are derived from the cumulative flow $N(x, t)$ as $\rho(x, t)=-\left.\frac{\Delta N}{\Delta x}\right|_{(x, t)}$ over $(x, t)$. The simulation time step $\Delta t$ for both methods is set to be one second. Under CTM, the road section is discretized into 28 cells which gives a spatial discretization $\Delta x$ to be 0.01786 (mile). The space-time discretization is set such that the CFL condition is satisfied for all fundamental diagrams (see Section 3.2 for details) adopted in the study. To maintain consistency, the density map under variational method are generated with the same spatial granularity. It is also worthy to note that the computational complexity of both methods are the same with the same space-time discretisation and fundamental diagrams adopted. The computational time of both methods are both linear in the number of time steps and number of locations 
considered. For each time and location point, both methods solve a minimisation problem (Equation (12) for CTM; Equation (18) for variational method). The only difference is that CTM solves for flows and densities while variational method solves for cumulative flows.

In the example, traffic is loaded into the link at saturation flow, $q_{\max }$, which is 1800 (veh/hr). The reason of using such high loading rate is to magnify the features of the numerical methods. The jam density $\bar{\rho}$ is set to be 240 (veh/mile). We first adopt a triangular fundamental diagram (Figure 5a) with which the speed of traffic under free-flow, $v$, is $30(\mathrm{mph})$ for all densities $\rho<\rho_{c}$. The critical density $\rho_{c}$ is $\frac{1800}{30}=60$ (veh $/ \mathrm{mile}$ ). The parameter $w$, which is the speed of the backward-propagating congestion, is taken as $10(\mathrm{mph})$ for all $\rho>\rho_{c}$. The red and green durations of the traffic light is set to be 30 (sec) and 30 (sec) respectively. During the green period, traffic is discharged at $q_{\max }$ and 0 (vph) during the red period. Consequently a queue is developed and propagates backward to the upstream end during red phase. As shown in Figure 3a, it is clear that numerical error (known as viscosity) arises along the density discontinuities under CTM, while the variational method (Figure 4a) is able to produce an exact solution.

We have to emphasize that the space-time discretizations used for both methods in the numerical examples are the same. The differences observed in Figures 3 and 4 are due to the solution methods themselves rather than the underlying discretization scheme. One may argue that the numerical error observed in CTM due to viscosity can be reduced by using finer space-time resolution, which is correct as discussed in LeVeque (1992). Of course, we should also know that improving numerical accuracy through refining resolution will have to come at the expense of computational effort. However, it is shown that the variational method with a triangular fundamental is indeed error-free (Daganzo and Menendez (2005); Daganzo (2006)) regardless of the space-time discretization used. Error will only arise in variational method when a more complicated fundamental diagram instead of a triangular one is used (Daganzo and Menendez, 2005).

\subsection{Platoon dispersion}

Figure 5 shows a set of fundamental diagrams with multi-segmented free-flow portions. An application of such construction is to generate different degree of dispersion of vehicle platoons or rarefaction waves (Geroliminis and Skabardonis, 2005). All fundamental diagrams shown in the figure have the same capacity $\left(q_{\max }=1800 \mathrm{veh} / \mathrm{hr}\right)$, critical density $\left(\rho_{c}=30 \mathrm{veh} / \mathrm{mile}\right)$, and jam density $(\bar{\rho}=240 \mathrm{veh} / \mathrm{mile})$. The difference between the fundamental diagrams lies on the free-flow portion: the two-segment (triangular) fundamental diagram (Figure 5a) has a free-flow portion with constant free-flow speed $(v=$ $30 \mathrm{mph}$ ) for all densities $\rho \in[0,60]$ (veh/mile); the three-segment fundamental diagram (Figure $5 \mathrm{~b}$ ) has a higher free-flow speed $\left(v_{1}=40 \mathrm{mph}\right)$ for densities $\rho \in[0,30]$ (veh $\left./ \mathrm{mile}\right)$ and a lower free-flow speed $\left(v_{2}=20 \mathrm{mph}\right)$ for densities $\rho \in[30,60]$ (veh/mile) to reflect a reduction in speed when traffic state gets close to the capacity; the four-segment fun- 
damental diagram (Figure $5 \mathrm{c}$ ) has three free-flow speeds $v_{1}=40 \mathrm{mph}, v_{2}=20 \mathrm{mph}$, and $v_{3}=10 \mathrm{mph}$ for densities $\rho \in[0,35]$ (veh/mile), $\rho \in[35,50]$ (veh/mile), and $\rho \in[50,60]$ (veh/mile) respectively; the five-segment fundamental diagram (Figure $5 \mathrm{~d}$ ) has four freeflow speeds $v_{1}=40 \mathrm{mph}, v_{2}=20 \mathrm{mph}, v_{3}=10 \mathrm{mph}$, and $v_{4}=5 \mathrm{mph}$ for densities $\rho \in[0,40]$ (veh/mile), $\rho \in[40,45]$ (veh/mile), $\rho \in[45,50]$ (veh/mile), and $\rho \in[50,60]$ (veh/mile) respectively. The shockwave speed $w$ is $-10 \mathrm{mph}$ in all cases.

It is noted these fundamental diagrams are all concave and hence they are all implementable under the variational framework. Figures 3 and 4 show the resulting density maps generated by CTM and variational method with different fundamental diagrams. Exact solutions are obtained for all these piecewise fundamental diagrams from the variational method which supports the findings in previous studies (Daganzo and Menendez (2005), Mazare et al. (2011)). With the multi-segmented free-flow part, portions of discharging traffic with different proceeding speeds and densities are generated. In addition to being used as a representation of the platoon dispersion phenomenon, this also gives further flexibility to the model for capturing traffic characteristics in the real world. Compared with the variational method, the density maps generated by CTM is distorted due to the errors arisen along the discontinuity between traffic states. Hence, we suggest that the variational formulation is a better numerical method for computing traffic dynamics with non-triangular fundamental diagrams.

To gain further insight, Figure 6 shows the discharging flow profiles estimated by the four fundamental diagrams in Figure 5 under the variational framework. The flow profiles are taken at a location $x_{1}=0.1$-mile downstream of the stopline. It is noted that the total traffic volumes under the flow profiles are all equal to 15 (veh) and hence traffic is conserved. The traffic signal turns green at time $t=0$ in the figure. With the triangular (two-segment) fundamental diagram, the discharged traffic takes 12 (sec) $(=0.1 /(30 / 3600))$ to reach $x_{1}$ where all traffic are considered to be proceeding with a common forward speed $30 \mathrm{mph}$. For the three-segmented fundamental diagram with two portion free-flow parts, the discharged traffic will reach $x_{1}$ in two packets. The first packet reaches $x_{1}$ at time $t=9(\mathrm{sec})(=0.1 /(40 / 3600))$ at speed $40(\mathrm{mph})$ and flow 1200 $(\mathrm{vph})(=40(\mathrm{mph}) \times 30(\mathrm{vpm}))$ as specified in the fundamental diagram. The second packet reaches $x_{1}$ at $t=18(\mathrm{sec})(=0.1 /(20 / 3600))$ at speed $20(\mathrm{mph})$ and flow 1800 (vph). Flow profiles can be derived for other fundamental diagrams in Figure 5 with similar method following the corresponding specifications of the fundamental diagrams. With the variational formulation, high quality solutions are obtainable even with the multi-segmented fundamental diagrams as shown in Figure 4. This enables us to easily capture the sophisticated but important feature of platoon dispersion phenomenon that cannot be modelled by triangular fundamental diagrams. A final note is that the wave-front tracking algorithms (e.g. Wong and Wong (2002); Henn (2005)) can also cope with platoon dispersion in a similar way as we use in the variational method. In fact, the wave-front tracking and the variational methods will come up with an identical solution if the same fundamental diagram is used. Nevertheless, as also noted by 
Mazare et al. (2011), the wave-front tracking algorithm is an event-based method which makes its computational complexity difficult to analyse. The algorithm becomes especially complicated when dealing with fundamental diagrams that have many pieces or even continuous. The variational method herein does not have such problem as it is implemented through a time-based approach.

\subsection{Moving bottlenecks (buses)}

Finally, we compare the performance of CTM and variational method on modelling moving bottlenecks. Here we consider a 1-unit long 4-lane arterial with saturation flow 7200 vph, jam density $480 \mathrm{vpm}$, free-flow speed $30 \mathrm{mph}$, and backward congestion propagation speed $-30 \mathrm{mph}$. The fundamental diagram here is considered to be triangular. Traffic is being loaded into the arterial at saturation flow $7200 \mathrm{vph}$. Suppose now a bus enters the arterial at location $x=0.3$ unit and time $t=0.01(\mathrm{hr})$. The bus proceeds with a speed $15 \mathrm{mph}$ and it stops at a bus stop at $x=0.6$ and $t=0.03$ (hr). The bus dwells at the stop for 2 minutes $(0.033 \mathrm{hr})$, then move on at previous speed and leaves the arterial at $x=0.9$ at $t=0.08(\mathrm{hr})$. The maximum passing rate (relative to the bus) of traffic around the bus is considered to be $5400 \mathrm{vph}$ (3 lanes out of 4 ) when the bus dwells at the bus stop and $2700 \mathrm{vph}$ when the bus moves at a slower speed relative to the surrounding traffic. Analytical solution can be derived for this simple example as described in Newell (1998). Numerically, the effect of this slow-moving bus can be captured in the variational framework through the cost formulation (22). For CTM, we adopt the method presented in Lebacque et al. (1998). To the best of our knowledge, Lebacque et al. (1998) still remains one of the very few documentation of modelling slow-moving buses on a kinematic wave platform. Following Lebacque et al. (1998), the movement of a bus over time and space is represented by a first-order kinematic law (i.e. distance travelled equals to integration of speed over time) and hence the simulator can track which cell the bus is in at each time step. The effect of the moving bottleneck induced by the bus is captured as follows: whenever the bus is proceeding more slowly than its surrounding traffic in the cell, the speed of 'all' traffic (no matter they are in front of or behind the bus) in the cell will be reduced to the speed of the bus.

Figures 7 and 8 respectively show the numerical result generated by variational method and CTM in which the arrowed solid line represents the trajectory of the bus on the space-time plane. We also include Figure 9 which shows the associated transition of traffic states along the fundamental diagram. Points A, B, C, and D in the figures respectively represent the events of the bus entering the arterial, arriving at the bus stop, leaving the bus stop, and leaving the arterial. Regions ' 1 ' refer to the traffic state ( $\rho=240(\mathrm{vpm}) ; q=7200(\mathrm{vph})$ ) before the bus enters the arterial. Region ' 2 ' is the traffic passing the slow-moving bus which is ( $\rho=180(\mathrm{vpm}) ; q=5400(\mathrm{vph}))$ as specified. Regions ' 3 ' are the traffic queued behind the bus while it is moving. The slope of the dotted line joining ' 2 ' and ' 3 ' in Figure 9 is the speed of the moving bus which is 15 (mph). Hence we can derive the flow and density at ' 3 ' from geometry as 6300 (vph) and 270 
(vpm) respectively. Region '4' is traffic state behind the bus when it is stopped. Region ' 4 ' has a flow value $5400 \mathrm{vph}$ and density 300 (vpm). Similar results can be derived from CTM as shown in Figure 8 while the dotted line pattern formed in Region ' 3 ' in Figure 8 is recognised as erroneous due to the uniform assumption (i.e. all traffic in the cell affected by the presence of the bus in the same way) adopted under CTM as discussed in Lebacque et al. (1998). Here we show that variational method provides a more accurate solution with respect to analytical one when dealing with moving bottleneck (buses).

\section{Real world application}

We now apply the solution methods to a real world scenario where we are looking at a 0.9-mile long section of Tottenham Court Road (TCR) in Central London, UK (Figure 10). The road section consists of two lanes and seven signal-controlled intersections. The traffic signals are operated under the SCOOT Urban Traffic Control system in which the cycle time, offsets, and green splits are adjustable according to real time traffic detection. Five of them: Bayley Street (N02/056), Goodge Street (N02/058), Torrington Place (N02/062), University Street (N02/060), and Grafton Way (N02/059), are equipped with loop detectors at which we have information of volumes and signal timings. The indices in the bracket are IDs of the junctions used under the SCOOT system. There are another two signal-controlled intersections: Howland Street and Store Street (marked by two triangles in the figure) at which there is no detection.

\subsection{Traffic data}

Traffic data used in this study include journey time estimates derived from the ANPR (Automatic Number Plate Recognition) system, which is an application of automatic vehicle identification (AVI) technique. We also have traffic flow and concentration measures collected from loop detectors in the city of London which are operated under the SCOOT urban traffic control (UTC) system. The data were collected on 5 June 2013 (Wednesday). With the journey times and loop detector data, we have the input information and benchmark for calibrating and validating our traffic models.

\subsubsection{Automatic vehicle identification}

A number of transport policies in London, including congestion charge and low emission zone schemes, are enforced by using the ANPR technology. The plate numbers of vehicles passing the ANPR cameras are recognized and recorded along with the corresponding times, which are used to decide whether the vehicles detected have paid the charge. The journey times of vehicles between two ANPR camera sites are then estimated by matching the license plate numbers. The journey times are processed and stored in 5-min averages. The journey time data can be used to derive various performance metrics such 
as such as speeds, journey time reliability, and impacts of major events including strikes (Tsapakis et al., 2012) and Olympics (Chow et al., 2014). This enables the road operator to calculate the benefits and costs associated with different policies or control plans to improve the day-to-day operation of the network. Nevertheless, it is noted that various errors may arise in matching the license plate numbers due to various reasons such as misreading of license plates, vehicles stopping en-route, and vehicles taking unusual long route between the two camera locations (Robinson and Polak, 2006). Consequently, a set of data filtering and processing rules is adopted to improve the journey time estimation. For example, the overtaking rules described in Robinson and Polak (2006) are used to eliminate the data noise caused by camera errors and delivery vehicles stopping along the route. Information from the Driver and Vehicle Licensing Agency (DVLA) is used to eliminate the data related to unauthorized vehicles on the bus lanes. In some occasions, data may be missing over some time intervals due to no sample (e.g. no vehicles can be matched during the time interval) or failure of hardware system. A range of patching algorithms will be used to impute the missing data in those circumstances.

\subsubsection{Urban loop detectors}

There are about 3,000 intersections in the city of London operating under the SCOOT Urban Traffic Control (UTC) system (Siemens, 2012). The intersections are equipped with loop detectors which measure the incoming flow and occupancy of traffic and hence derive optimal timing strategies in real-time. For performance measure purpose, measured traffic quantities including flows and occupancies are stored and processed in archived SCOOT dataset. The dataset also records signal timings used, excessive queues detected, and journey times estimated. These information are stored in different 'messages' in the SCOOT dataset and different messages record different traffic characteristics and estimates (Siemens, 2012). The SCOOT dateset provides the following information of demand and signal timings through its 'M13' and 'M29' messages (Siemens, 2012):

1. Flow counts - flow counts are recorded once a signal cycle at each SCOOT detector station in the M29 messages. The cycle time in the TCR area is 88 -sec and hence the unit of these flow counts will be [veh/88-sec].

2. Signal timings (green durations) - SCOOT M13 messages provide the durations of green phases. The green durations are time-varying and derived from the SCOOT optimiser in real time.

\subsection{Building traffic models}

In addition to demands, signal timings, and split ratios as stated in previous section, we need to determine the fundamental diagram of each link in order to complete the specification of the traffic models. The key parameters here are wave speeds, saturation 
flows, critical and jam densities. First, the SCOOT detector data are processed and stored in averages over a signal cycle with which we cannot identify the true values of saturation flow with these cyclic averages. Our way to estimate the saturation flow is to assume the maximum cyclic flow value observed on an approach will be equal to $\left(\frac{g}{g+r} s\right)$, where $g$ and $r$ are respectively the effective green and red durations allocated to that approach over a cycle, and $s$ is the saturation flow which is an unknown. In traffic engineering, this $\left(\frac{g}{g+r} s\right)$ is regarded as the signal-controlled capacity of the approach. It is noted that both $g$ and $r$ are records of real-time operations. However, both $g$ and $r$ are not constant due to the adaptive nature of SCOOT controller and hence we are taking the average values of them when calibrating the traffic model. The sum of them $(g+r)$ gives the total cycle time $c$ which is 88 -sec in the TCR network. Consequently, the saturation flow $s$ on each approach can be derived accordingly.

Table 1 summarises the measured maximum flows (in [vph per lane]) in one cycle and average $\left(\frac{g}{g+r}\right)$ ratios allocated to TCR during the study period at the four intermediate detector stations (N02/058, N02/062, N02/060, and N02/059). The table also shows the corresponding estimated saturation flows $s$. To further investigate the reliability of the saturation flow estimation, we conduct a sensitivity analysis on the corresponding overall journey time and flow estimates produced by the model with respect to different choices of $s$ values. As an example, Figure 11 shows the sensitivity of journey time and flow estimation error with respect to the saturation flow $s$ at Junction Goodge Street (N02/058) over a range of values from $1400 \mathrm{vph} /$ lane - $1800 \mathrm{vph} /$ lane. The errors are measured in terms of Mean-Absolute-Percentage-Error (see Equation 23). It is shown that a saturation flow of around $1550 \mathrm{vph} /$ lane will give the lowest error for journey time and flow estimations. This $1550 \mathrm{vph} /$ lane indeed is consistent with the number we obtain in Table 1. Similar results are observed at the other three junctions and hence it suggests using the controlled capacity ' $\left(\frac{g}{g+r} s\right)$ ' is a reasonably reliable way to estimate saturation flows with coarse data. Finally, we note that the estimated values in Table 1 appear to be less than the nominal value 1800 vph per lane. We reckon this is due to the narrow streets, high volumes of turning traffic, and pedestrian crossing in the area.

Moreover, SCOOT dataset does not provide a reliable estimate of jam density. To facilitate the calibration process, here we adopt a nominal value: $225 \mathrm{veh} / \mathrm{mile}$ per lane. Given the saturation flows and jam densities, the main objective of calibration now is to determine the wave speeds $\mathbb{U}$ with which we can specify the fundamental diagrams accordingly. The wave speeds are determined such that the discrepancies between the journey times along TCR derived from the traffic models and those measured from the on-site ANPR system are minimised. For CTM, the journey times are derived by using the frozen field method (Chow et al., 2010). After running a CTM simulation, one can obtain the traffic speed matrix $v_{i}(t)=\frac{q_{i}(t)}{\rho_{i}(t)}$ over all cells $i$ and times $t$. We can then 'release' a virtual infinitesimal probe at the upstream end of the route over a set of departure times $\sigma$. Using basic principle of kinematics, the distance travelled of each of 
these probes over time can be obtained from integrating the speed $v_{i}(t)$ over time and space. The probe is said to be exiting the current cell $i$ and entering the subsequent cell $i+1$ at time $\sigma_{1}$ when the distance travelled is greater than or equal to $\Delta x_{i}$. The travel time of the probe through cell $i$ is then determined as $\tau_{i}=\left(\sigma_{1}-\sigma\right)$. Applying the same methodology to other cells and departure times, a travel time profile through the entire route can be derived accordingly. For variational method, the trajectory of vehicles can be derived as the iso-contours of the $N$-values (Daganzo and Menendez, 2005), which can give the corresponding journey time of each vehicle.

After specifying the general form of the fundamental diagrams (i.e. the number of segments and where the fundamental diagrams are segmented), we use iterative line searches to estimate the corresponding wave speeds in the fundamental diagrams aiming to minimise the discrepancies between modelled and measured journey times. The results are shown in Figure 12. All data are collected on 5 June 2013 (Wednesday) from 12:00 - 15:00. Figure 12a are estimates produced by models with two-segment fundamental diagrams while Figure $12 \mathrm{~b}$ are estimates produced with three-segment fundamental diagrams. In both figures, the dotted lines as 'ANPR' are measured journey times from ANPR system and they are regarded as 'ground truth' here. 'CTM' are journey times estimated by CTM and 'VM' are journey times derived from the variational method. With the two-segment fundamental diagrams, both CTM and the variational method have a tendency of underestimating the journey times through neglecting the reduction in speed when traffic approaching high values of density. With the three-segment fundamental diagrams, the feature of platoon dispersion can be captured (see Figure 6 ) which enables traffic proceeding under free-flow at different density to be associated with different speed. Consequently, traffic will slow down when the associated density grows toward the critical value under the three-segment fundamental diagram specification. The resultant model fits better with reality and also gives additional flexibility in parameter specification. Hence the model can produce more accurate estimates of journey times with respect to the observed values. Nevertheless, one should note that there can be other factors contributing to the underestimation of journey times. This includes oversimplification of the junction dynamics, presence of pedestrians, and heterogeneity of traffic (e.g. presence of slow moving vehicles such as buses, bikes, etc). Incorporating these factors require more advanced modelling techniques such as detailed node modelling that captures the discrete movements of vehicles at junctions, and multiclass modelling that considers explicitly the heterogeneity of traffic flow. These will be beyond the scope of the present paper however and we will leave these to future study.

To gain a quantitative picture, we calculate the Mean-Absolute-Percentage-Error (MAPE) of journey times $\epsilon_{\tau}$ estimates with respect to the observed values as:

$$
\epsilon_{\tau}=\frac{1}{T} \sum_{t=0}^{T}\left|\frac{\hat{\tau}(t)-\tau(t)}{\tau(t)}\right|,
$$


where $\hat{\tau}(t)$ and $\tau(t)$ are respectively the estimated and measured journey times at time interval $t$ within the time horizon $T$. The MAPEs of estimates produced by CTM (2segment), CTM (3-segment), the variational method (2-segment), and the variational method (3-segment) are $15.3 \%, 15.2 \%, 15.6 \%$, and $14.6 \%$ respectively. The differences between the estimates of CTM and the variational method are rather insignificant. Nevertheless, it is interesting to note that the variational method gives a slightly better improvement compared with CTM when a three-segmented fundamental diagram is adopted. We believe it is because of the numerical errors in CTM at the density discontinuities are accumulated with the increased overlapping different traffic states when a multi-segment fundamental diagram is adopted. We also explore the use of more refined fundamental diagrams, where we obtain error rates of around $14 \%$ for four- and fivesegmented fundamental diagrams. The error rates are lower than that obtained with three-segment fundamental diagram while they are not significantly better despite the additional computational effort. Hence, we conclude that the three-segment fundamental diagrams will be a good enough representation of traffic characteristics in this context.

To gain further insight on the performance of the models, Figures 13 and 14 respectively show the corresponding cyclic flows estimated by CTM and the variational method with three-segment fundamental diagrams at the four detector stations (N02/058, N02/062, $\mathrm{N} 02 / 060$, and N02/059). We compare these estimated flow values against the actual observed values by the detectors. The error rates (in terms of MAPEs) at each station are summarised is Table 2 which shows that the flow estimates by CTM and variational method are close. The average MAPE of the flow estimates of the variational method is $14.3 \%$ while that of CTM is $14.8 \%$. This should not be surprising as the main different between variational method and CTM is the viscosity in traffic estimates. Nevertheless, when the traffic quantities are aggregated to reasonably long time scale (say, one signal cycle here), the discrepancy due to such viscosity effect will be averaged out and hence the two methods will produce similar results. Further improvement in flow estimation will require detector data with higher quality and spatio-temporal granularity which are unfortunately not available for the present study. Hence, we will leave them here for future exploration. Finally, it is noted that we do not include 'moving bottleneck' herein due to the lack of appropriate data such as bus trajectories and surrounding traffic states. Meanwhile we are contacting different authorities around the world in order to get required data. We wish to report further developments in the future.

\section{Concluding remarks}

This paper presents a variational based modelling framework of urban traffic dynamics. We compare this variational method with the traditionally used CTM over a set of hypothetical experiments and a real case in Central London. The numerical experiments suggest that the variational method is easy to implement and able to produce high quality solution, in particular for modelling platoon dispersions and moving bottle- 
necks. The case study with Central London data supports the validity of the variational method for real world applications. A note to emphasize is that this paper is not aiming to claim variational method is a better 'model' than CTM, as theoretically they are both belong to same model - LWR model. The results are only showing variational method is a more accurate solution method to LWR model than CTM or Godunov which echoes the findings in Daganzo (2005b) and Daganzo and Menendez (2005). It also suggests variational method can be a better implementation than CTM when dealing with platoon dispersions and moving bottlenecks. One may argue that the smeared solution (i.e. solution with viscosity) produced by CTM and other Godunov schemes may indeed be a better representation of actual traffic. We also agree that the sharp jump discontinuity is only a feature of LWR model and is not a real world feature. However, we think that the viscosity is indeed an unexpected characteristics due to the property of the underlying solution method. Indeed the LWR model itself does not explicitly specify such viscosity in its formulation. We believe a good solution method should simply produce a numerical solution as close to the exact or theoretical solution as possible. Whether the numerical solution is a good representation of reality should be a separated question. If one is interested in producing a solution that captures the viscosity, perhaps one should consider revising the model formulation or using a different model rather than LWR.

It is known that as first order model the LWR framework cannot capture traffic dynamics associated with acceleration and deceleration. Nevertheless, modelling acceleration and deceleration is an important issue for evaluating a number of performance metrics including fuel consumption and emission. Extension of the current modelling framework, including consideration of spatial and temporal resolutions, for capturing these higher order traffic dynamics will be an important future study. Moreover, there have been studies (e.g. Mehran et al. (2012), Han et al. (2014)) exploring the development of practical estimation and optimisation algorithms based upon the variational method. Considering the benefits of the variational method revealed herein, we believe the variational method will be an useful and computationally effective tool for estimating and managing urban traffic and hence should receive more attention in the research community. As an extension of Chow and Lo (2007), we are currently investigating the sensitivity of traffic state estimates with respect to various perturbations including control schemes, incidents, and measurement errors based upon the variational formulation. Following Chow and $\mathrm{Li}$ (2014), we are also developing a variational based stochastic modelling framework with applications of a recently proposed set-valued fundamental diagrams (Kurzhanskiy and Varaiya, 2012). With the sensitivity analysis and stochastic formulation, efficient and robust control can be derived which not just minimises the travel delay, but also minimises the associated variability.

\section{Acknowledgements}

The authors would like to thank the anonymous referees for their constructive com- 
ments. The study is funded by the China Scholarship Council (CSC) and UCL Faculty of Engineering. The authors would also like to acknowledge UK Transport for London (TfL) for providing the ANPR and SCOOT data. The contents of this paper reflect the views of the authors who are responsible for the facts and the accuracy of the data presented herein. The contents do not reflect the official views or policies of Transport for London or any other organisations.

\section{References}

[1] Balijepallia, N. C., Ngoduy, D. and Watling, D. (2013), 'The two-regime transmission model for network loading in dynamic traffic assignment problems', Transportmetrica A 1(1), 1-22.

[2] Chow, A. H. F. (2009), 'Dynamic system optimal traffic assignment - a statedependent control theoretic approach', Transportmetrica 5(2), 85-106.

[3] Chow, A. H. F., Dadok, V., Dervisoglu, G., Gomes, G., Horowitz, R., Kurzhanskiy, A., Kwon, J., Lu, X., Muralidharan, A., Norman, S., Sanchez, R. and Varaiya, P. (2008), 'TOPL: Tools for operational planning of transportation networks', Proceedings of the 1st ASME Dynamic Systems and Control Conference (CD-ROM), October 20-22. Ann Arbor, MI, USA.

[4] Chow, A. H. F., Gomes, G., Kurzhanskiy, A. A. and Varaiya, P. (2010), 'Aurora RNM - a macroscopic tool for arterial traffic analysis', Proceedings of 89th Annual Meeting of Transportation Research Board. Washington, DC, USA .

[5] Chow, A. H. F. and Li, Y. (2014), 'Robust optimization of dynamic motorway traffic via ramp metering', IEEE Transactions on Intelligent Transportation Systems 15(3), 1374-1380.

[6] Chow, A. H. F. and Lo, H. (2007), 'Sensitivity analysis of signal control with physical queuing: delay derivatives and an application', Transportation Research Part B 41(3), 462-477.

[7] Chow, A. H. F., Santacreu, A., Tsapakis, I., Tanasaranond, G. and Cheng, T. (2014), 'Empirical assessment of urban traffic congestion', Journal of Advanced Transportation 48(8), 1000-1016.

[8] Courant, R., Friedrichs, K. and Lewy, H. (1928), 'Über die partiellen Differenzengleichungen der mathematischen Physik', Mathematische Annalen 100, 32-74.

[9] Daganzo, C. F. (1994), 'The cell transmission model: A dynamic representation of highway traffic consistent with the hydrodynamic theory', Transportation Research Part B 28(4), 269-287. 
[10] Daganzo, C. F. (1995), 'The cell-transmission model, part II: network traffic', Transportation Research Part B 29(2), 79-93.

[11] Daganzo, C. F. (2005a), 'A variational formulation of kinematic waves: basic theory and complex boundary conditions', Transportation Research Part B 39(2), 187-196.

[12] Daganzo, C. F. (2005b), 'A variational formulation of kinematic waves: solution methods', Transportation Research Part B 39(10), 934-950.

[13] Daganzo, C. F. (2006), 'On the variational theory of traffic flow: well-posedness, duality and applications', Networks and heterogeneous media 1(4), 601-619.

[14] Daganzo, C. F. and Menendez, M. (2005), 'A variational formulation of kinematic waves: bottleneck properties and examples', In: Transportation and Traffic Flow Theory pp. 345-364.

[15] Geroliminis, N. and Skabardonis, A. (2005), 'Prediction of arrival profiles and queue lengths along signalized arterials by using a markov decision process', Transportation Research Record 1934, 116-124.

[16] Godunov, S. K. (1959), 'A difference method for numerical calculations of discontinuous solutions of the equations of hydrodynamics', Matematicheskii Sbornik 47, 271-306.

[17] Gomes, G. and Horowitz, R. (2006), 'Optimal freeway ramp metering using the asymmetric cell transmission model', Transportation Research Part C 14(4), 244 262.

[18] Han, K., Friesz, T. and Yao, T. (2013a), 'A partial differential equation formulation of vickreys bottleneck model, part I: Methodology and theoretical analysis', Transportation Research Part B 49, 55-74.

[19] Han, K., Friesz, T. and Yao, T. (2013b), 'A partial differential equation formulation of vickreys bottleneck model, part II: Numerical analysis and computation', Transportation Research Part B 49, 75-93.

[20] Han, K., Gayah, V., Piccoli, B., Friesz, T. and Yao, T. (2014), 'On the continuum approximation of the on-and-off signal control on dynamic traffic networks', Transportation Research Part B 61, 73-97.

[21] Henn, V. (2005), 'Tracking waves through spatial discontinuities: boundary conditions in the wave tracking resolution of the lwr model', Mathematics in Transport pp. 349-362.

[22] Heydecker, B. G. and Addison, J. D. (2005), 'Analysis of dynamic traffic equilibrium with departure time choice', Transportation Science 39(1), 39-57.

[23] Jabari, S. E. and Liu, H. X. (2012), 'A stochastic model of traffic flow: theoretical foundations', Transportation Research Part B 46, 156-174. 
[24] Jin, W., Chen, L. and Puckett, E. (2009), 'Supply-demand diagrams and a new framework for analyzing the inhomogeneous Lighthill-Whitham-Richards model', Transportation and Traffic Theory pp. 603-635.

[25] Kurzhanskiy, A., Kwon, J. and Varaiya, P. (2009), 'Aurora road network modeler', Proceedings of the 12th IFAC Symposium on Control in Transportation Systems (CD-ROM), September 2-4. Redondo Beach, CA, USA .

[26] Kurzhanskiy, A. and Varaiya, P. (2012), 'Guaranteed prediction and estimation of the state of a road network', Transportation Research Part C 21, 163-180.

[27] Laval, J. (2004), 'Hybrid models of traffic flow: Impacts of bounded vehicle accelerations', PhD thesis, University of California Berkeley .

[28] Laval, J. A. and Leclercq, L. (2013), 'The Hamilton-Jacobi partial differential equation and the three representations of traffic flow', Transportation Research Part B 52, 17-30.

[29] Lebacque, J. P., Lesort, J. B. and Giorgi, F. (1998), 'Introducing buses into firstorder macroscopic traffic models', Transportation Research Record 1644, 70-79.

[30] LeVeque, R. J. (1992), Numerical methods for conservation laws, Birkhauser Verlag.

[31] Lighthill, M. J. and Whitham, G. B. (1955), 'On kinematic waves. i. flood movement in long rivers', Proceedings of the Royal Society of London. Series A. Mathematical and Physical Sciences 229(1178), 281-316.

[32] Lo, H. and Szeto, W. Y. (2002), 'A cell-based variational inequality formulation of the dynamic user optimal assignment problem', Transportation Research Part B 36(5), 421-443.

[33] Mazare, P., Dehwah, A., Claudel, C. G. and Bayen, A. M. (2011), 'Analytical and grid-free solutions to the Lighthill-Whitham-Richards traffic flow model', Transportation Research Part B 45, 1727-1748.

[34] Mehran, B., Kuwahara, M. and Naznin, F. (2012), 'Implementing kinematic wave theory to reconstruct vehicle trajectories from fixed and probe sensor data', Transportation Research Part C 20, 144-163.

[35] Newell, G. F. (1993), 'A simplified theory of kinematic waves in highway traffic: I: general theory, II: queuing at freeway bottlenecks, III: multi-destination flows', Transportation Research Part B 27, 281-313.

[36] Newell, G. F. (1998), 'A moving bottleneck', Transportation Research Part B 32, 531-537.

[37] Nie, Y., Ma, J. and Zhang, M. H. (2008), 'A polymorphic dynamic network loading model', Computer-aided Civil and Infrastructure Engineering 23, 86-103. 
[38] Richards, P. I. (1956), 'Shock waves on the highway', Operations Research 4(1), pp. $42-51$.

[39] Robinson, S. and Polak, J. (2006), 'Overtaking rule method for the cleaning of matched license plate data', ASCE Journal of Transportation Engineering 132(8), 609-617.

[40] Siemens (2012), 'SCOOT user guide', Siemens Mobility, Traffic Solutions UTC System .

URL: www.siemens.co.uk/traffic/pool/downloads/handbooks/utc/

[41] Szeto, W. Y. (2008), 'The enhanced lagged cell transmission model for dynamic traffic assignment', Transportation Research Record 2085, 76-85.

[42] Szeto, W. Y. and Lo, H. (2006), 'Dynamic traffic assignment: properties and extensions', Transportmetrica $\mathbf{2}, 31-52$.

[43] Tsapakis, I., Turner, J., Cheng, T., Heydecker, B. G., Emmonds, A. and Bolbol, A. (2012), 'Effects of tube strikes on journey times in the transport network of london', Transportation Research Record 2274, 84-92.

[44] Vickrey, W. S. (1969), 'Congestion theory and transport investment', American Economics Review 59, 251-261.

[45] Wong, S. C. and Wong, G. C. K. (2002), 'An analytical shock-fitting algorithm for lwr kinematic wave model embedded with linear speed-density relationship', Transportation Research Part B 36(8), 683-706.

[46] Yperman, I. (2007), 'The link transmission model for dynamic network loading', PhD thesis, Katholieke Universiteit Leuven, Belgium .

[47] Zhang, H., Nie, Y. and Qian, Z. (2013), 'Modelling network flow with and without link interactions: The cases of point queue, spatial queue and cell transmission model', Transportmetrica B 1(1), 33-51.

[48] Zhu, F., Lo, H. and Lin, H. Z. (2013), 'Delay and emissions modeling for signalized intersection', Transportmetrica B 1(2), 111-135.

[49] Ziliaskopoulos, A. (2000), 'A linear programming model for the single destination system optimum dynamic traffic assignment problem', Transportation Science 34(1), $37-49$. 
Table 1: Estimation of saturation flows on Tottenham Court Road (TCR)

\begin{tabular}{|l|cccr|}
\hline & N02/058 & N02/062 & N02/060 & N02/059 \\
\hline $\begin{array}{l}\text { Max. flow } \\
\text { (vph per lane) }\end{array}$ & 655 & 675 & 634 & 695 \\
\hline g/(g+r) & 0.42 & 0.43 & 0.41 & 0.43 \\
\hline $\begin{array}{l}\text { Saturation flow } \\
\text { (vph per lane) }\end{array}$ & 1554 & 1572 & 1555 & 1620 \\
\hline
\end{tabular}

Table 2: Flow errors of different solution schemes

\begin{tabular}{|l|rrrr|r|}
\hline & N02/058 & N02/062 & N02/060 & N02/059 & Average \\
\hline CTM & $10.14 \%$ & $16.54 \%$ & $15.70 \%$ & $16.80 \%$ & $14.80 \%$ \\
VM & $9.53 \%$ & $17.05 \%$ & $14.83 \%$ & $15.81 \%$ & $14.30 \%$ \\
\hline
\end{tabular}




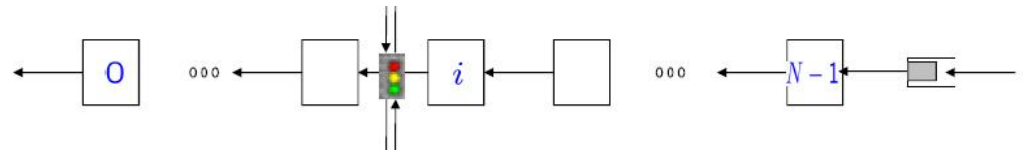

Figure 1: Cell representation of a signal-controlled link

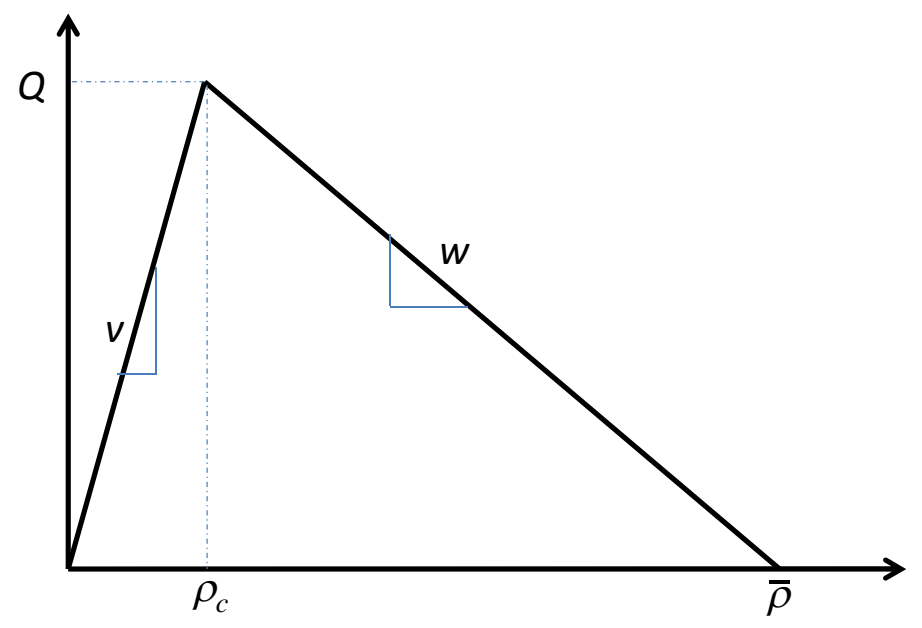

Figure 2: Triangular fundamental diagram in CTM (Daganzo, 1994) 

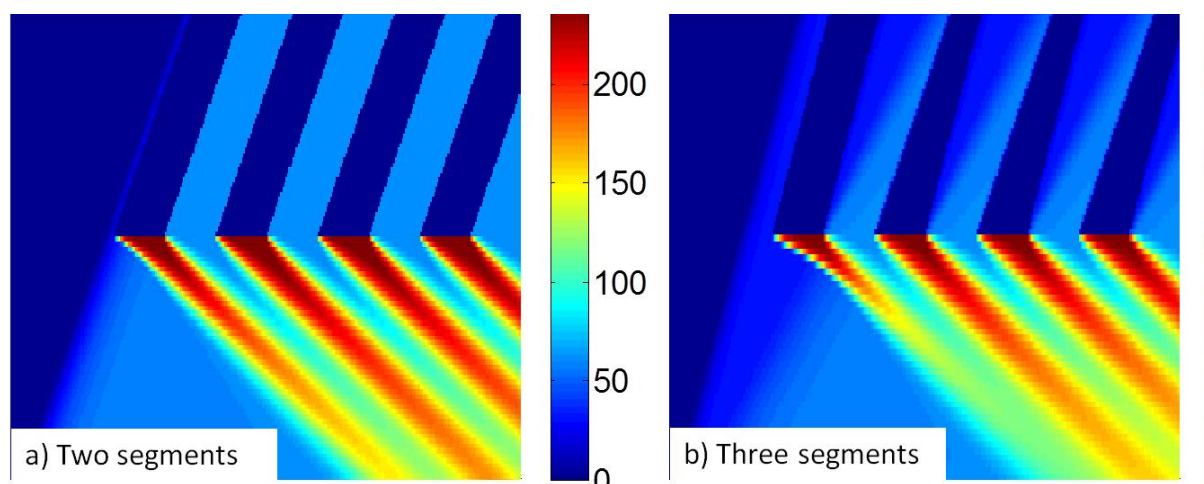

200

150
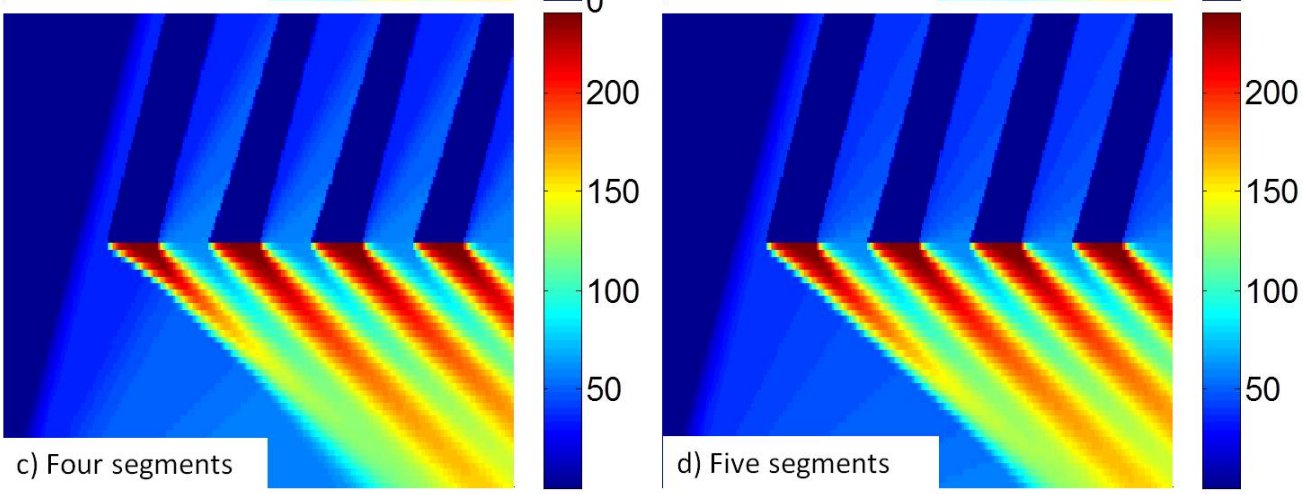

Figure 3: Density maps (in: [veh/mile]) generated by CTM with different fundamental diagram specifications 


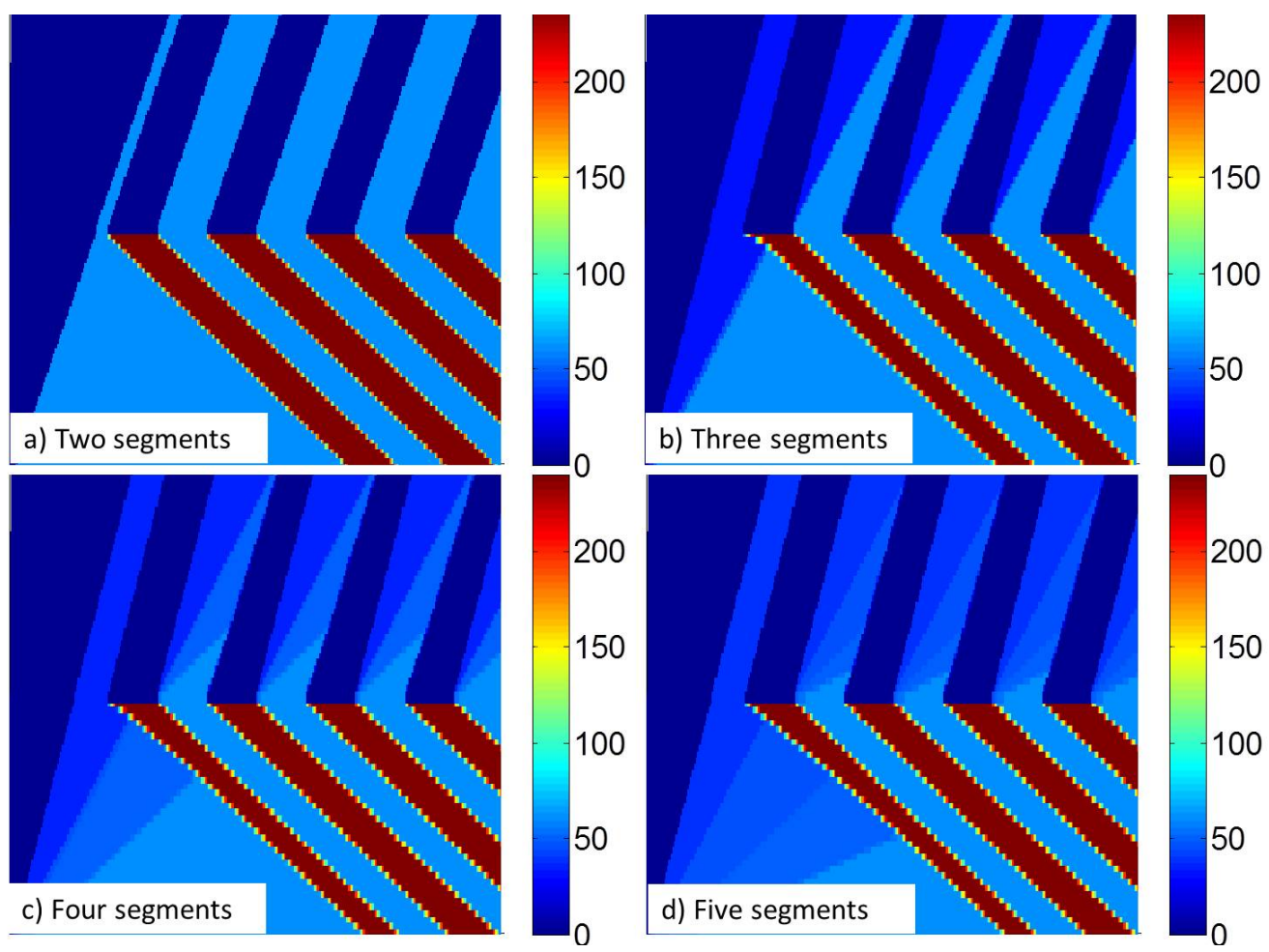

Figure 4: Density maps (in: [veh/mile]) generated by variational method with different fundamental diagram specifications 

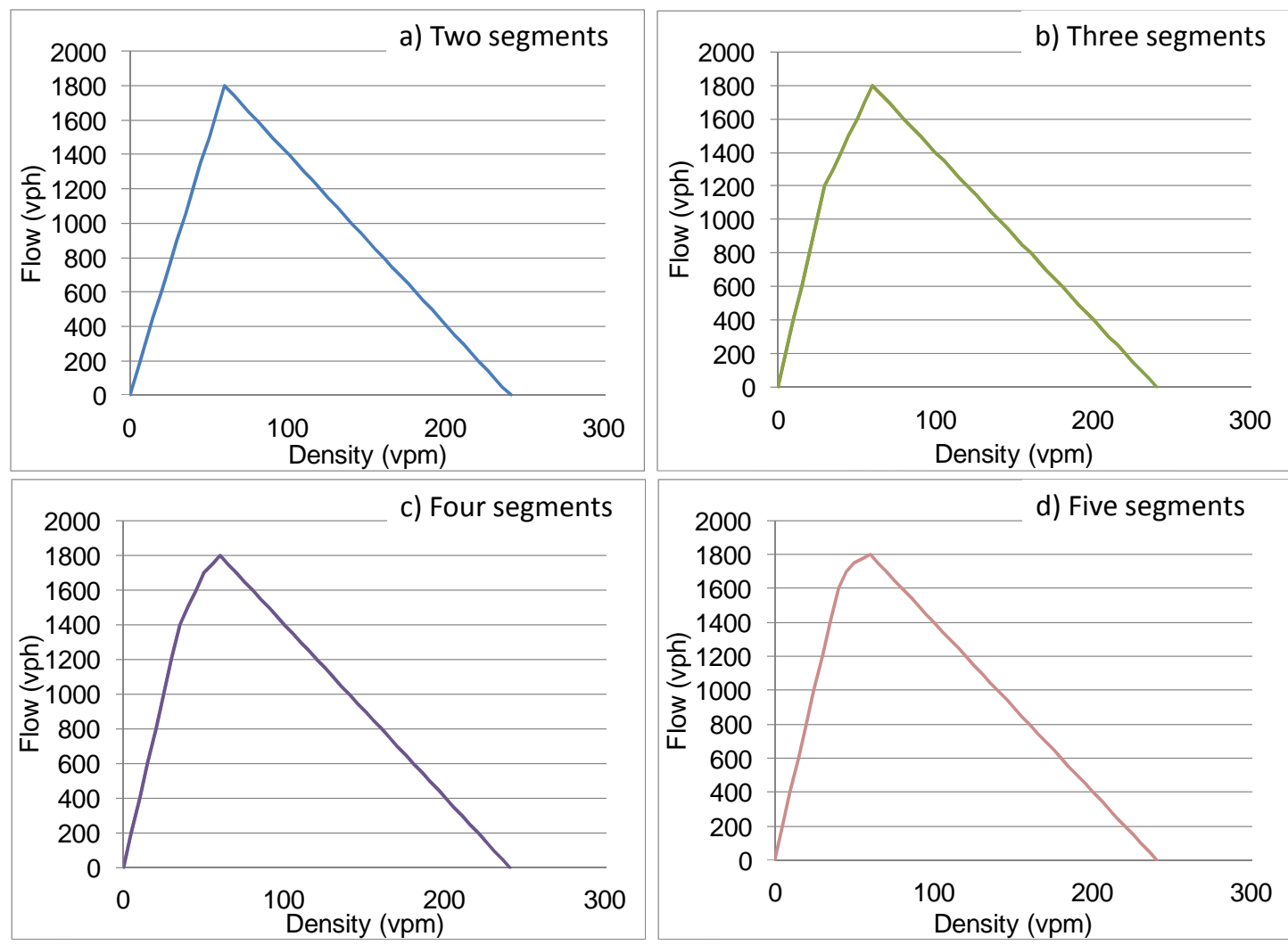

Figure 5: Fundamental diagrams with different numbers of segments

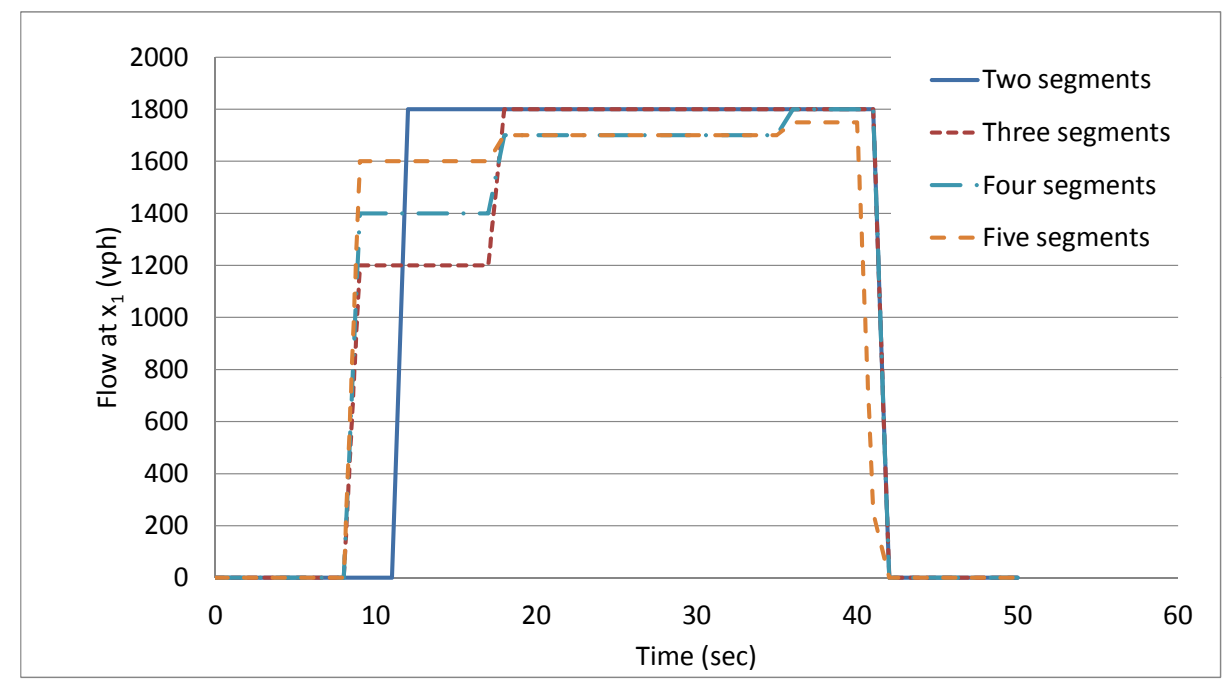

Figure 6: Outflow profiles generated with different fundamental diagram specifications 


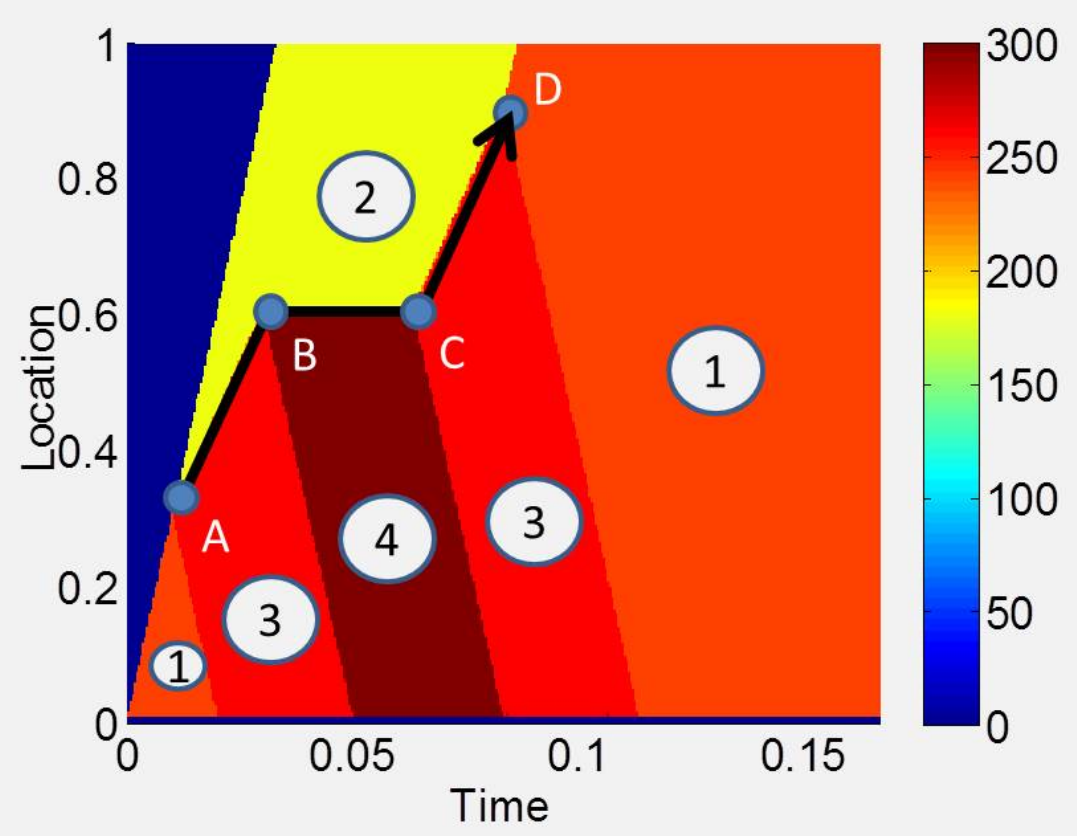

Figure 7: Simulation of impact of a slow-moving bus under variational representation

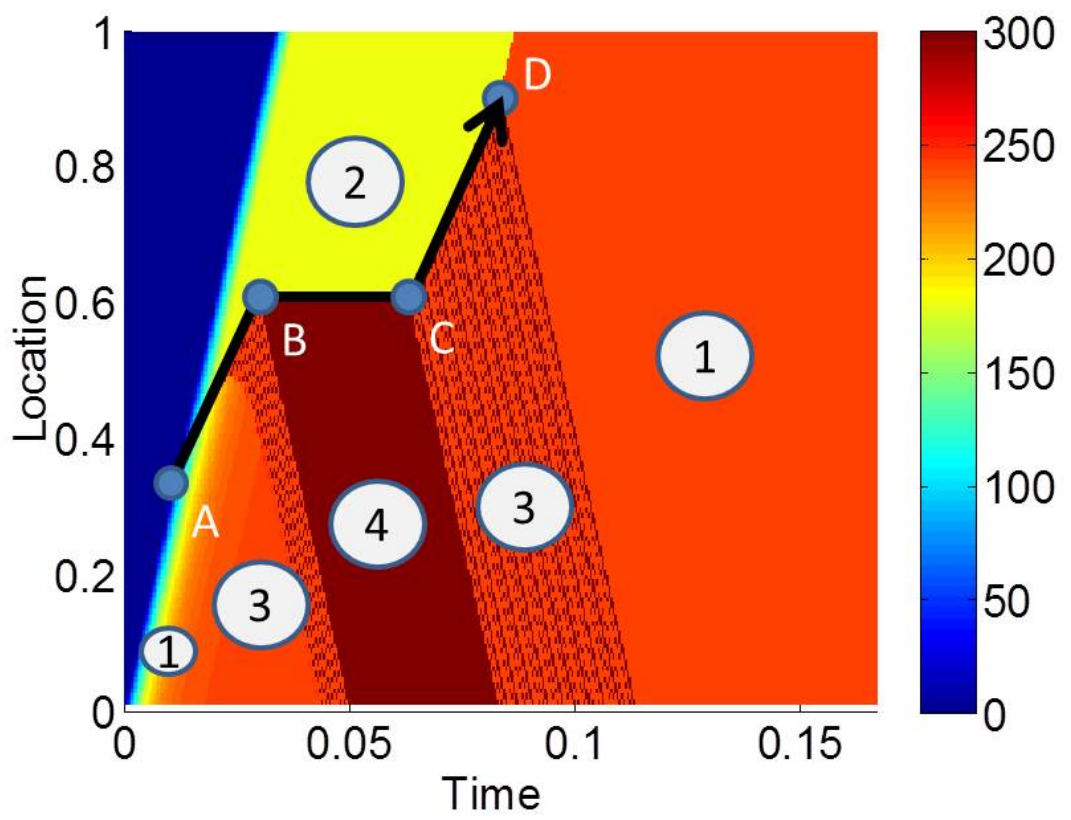

Figure 8: Simulation of impact of a slow-moving bus under CTM representation 


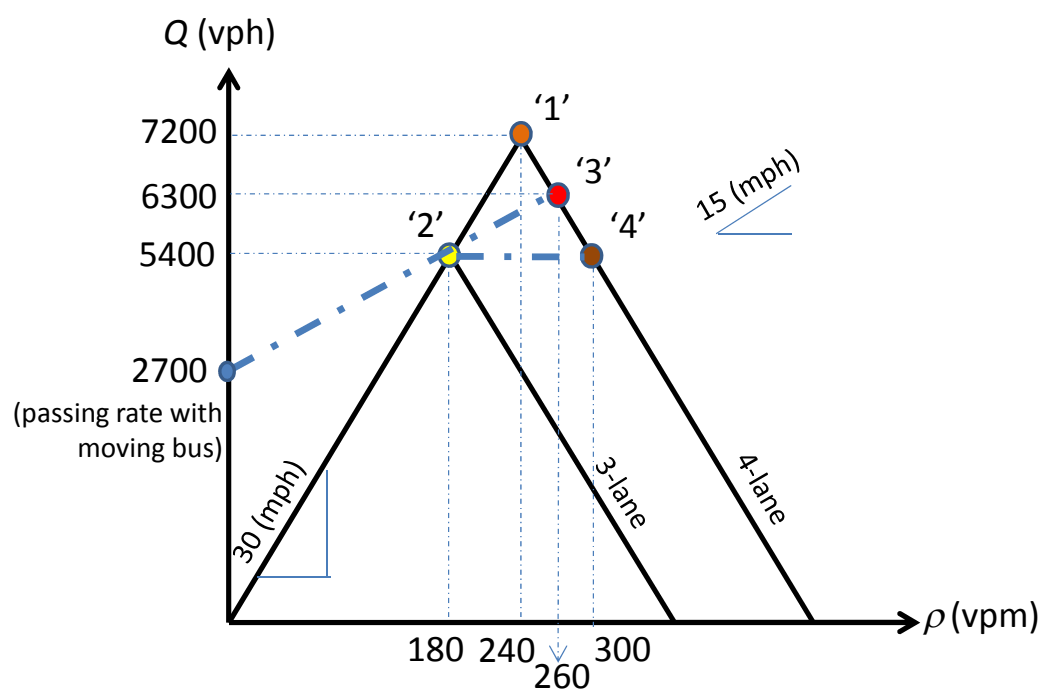

Figure 9: Transition of traffic states associated with the slow-moving bus 


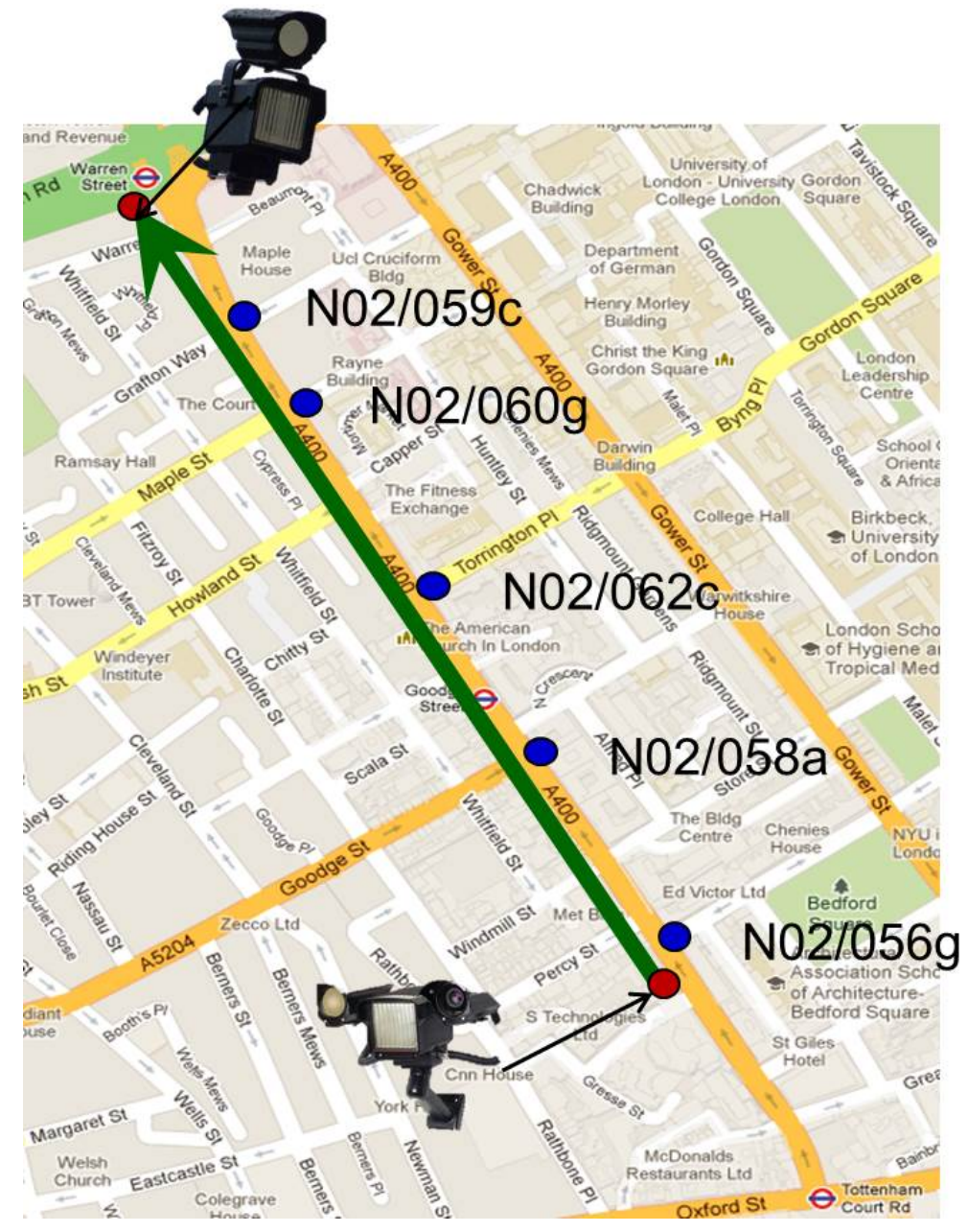

Figure 10: Tottenham Court Road (TCR), London, UK 

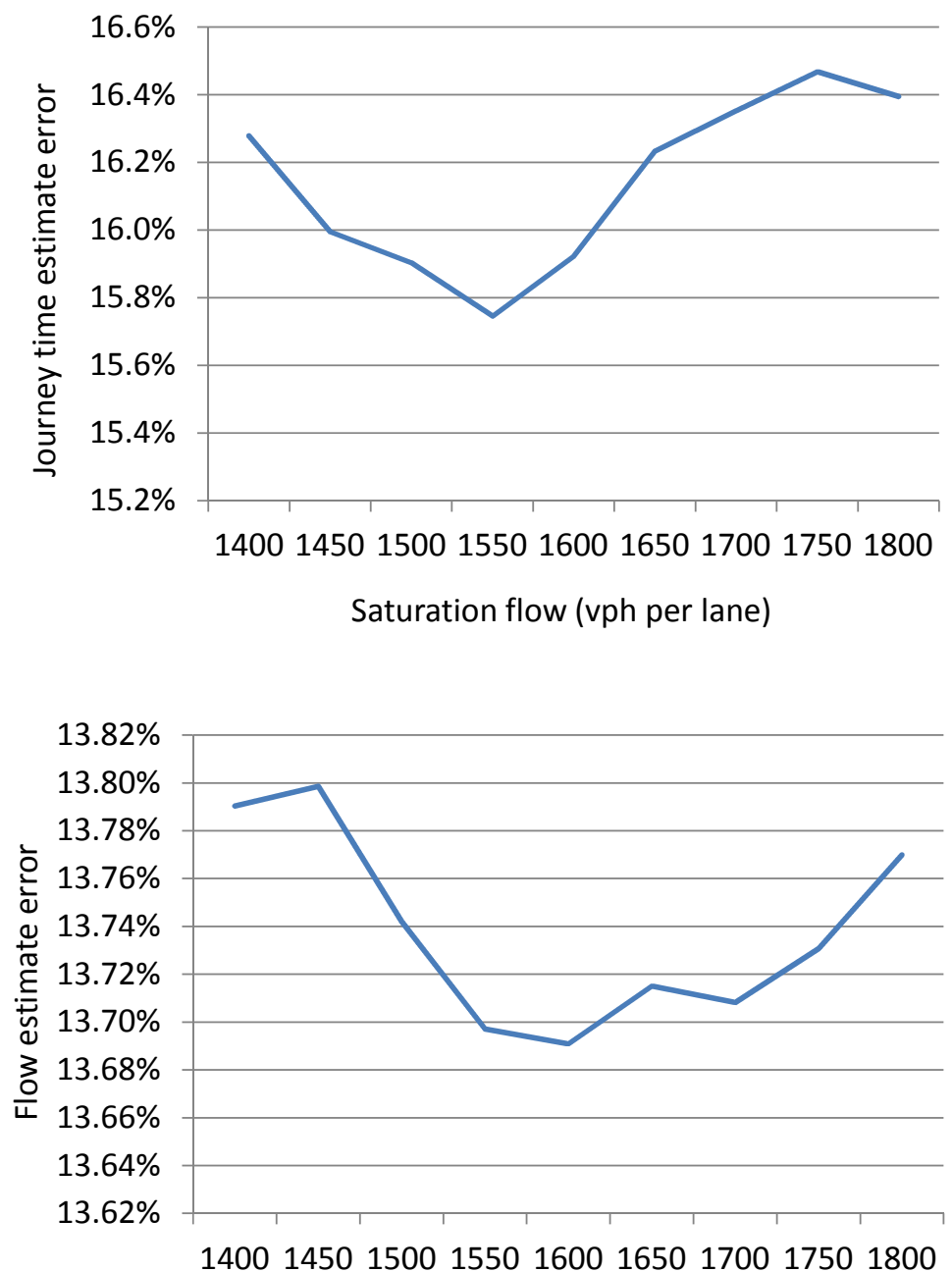

Saturation flow (vph per lane)

Figure 11: Sensitivity analysis of estimation errors with respect to choice of saturation flow (at Goodge Street) 

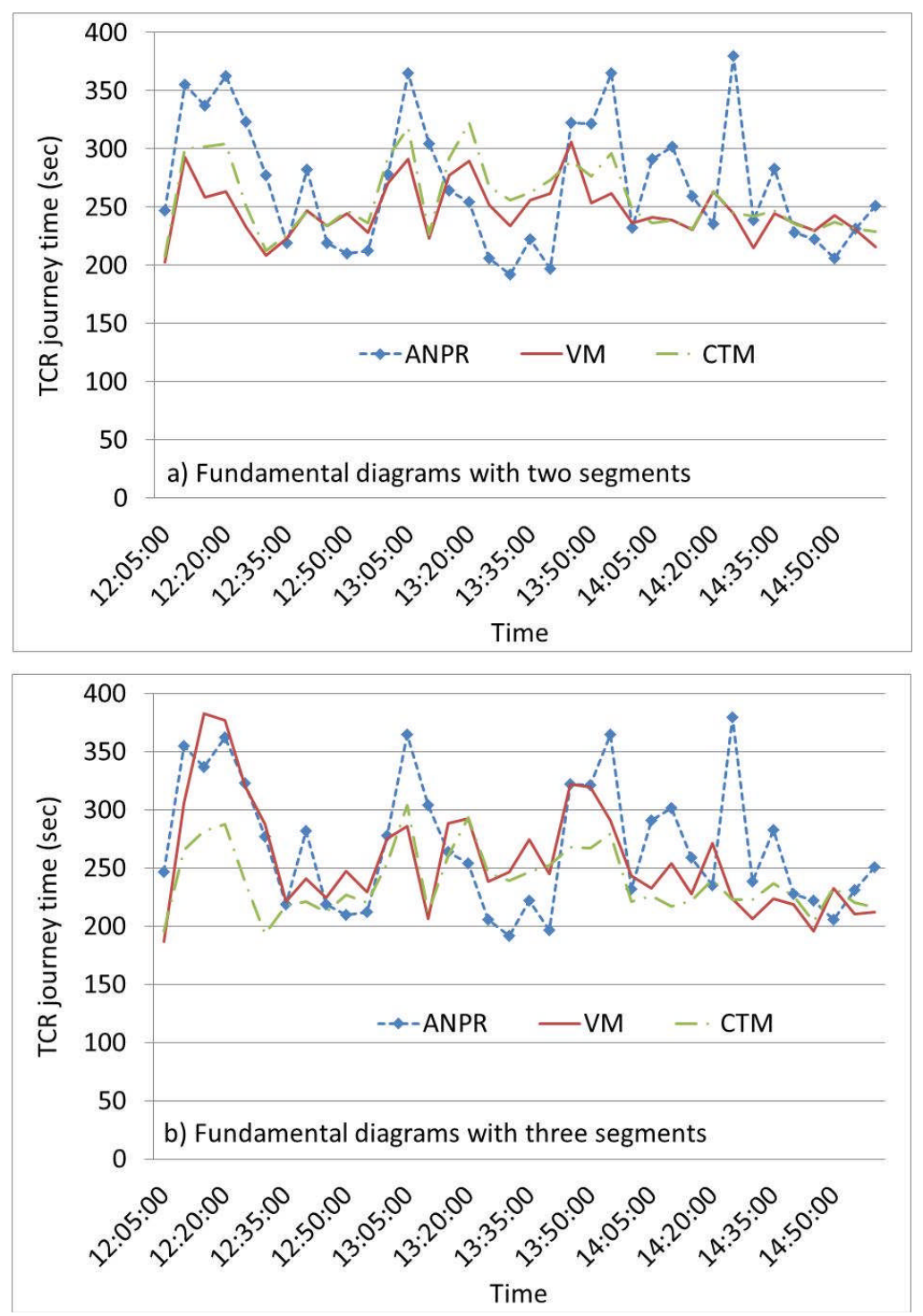

Figure 12: Comparison of TCR journey times (5 June 2013) 


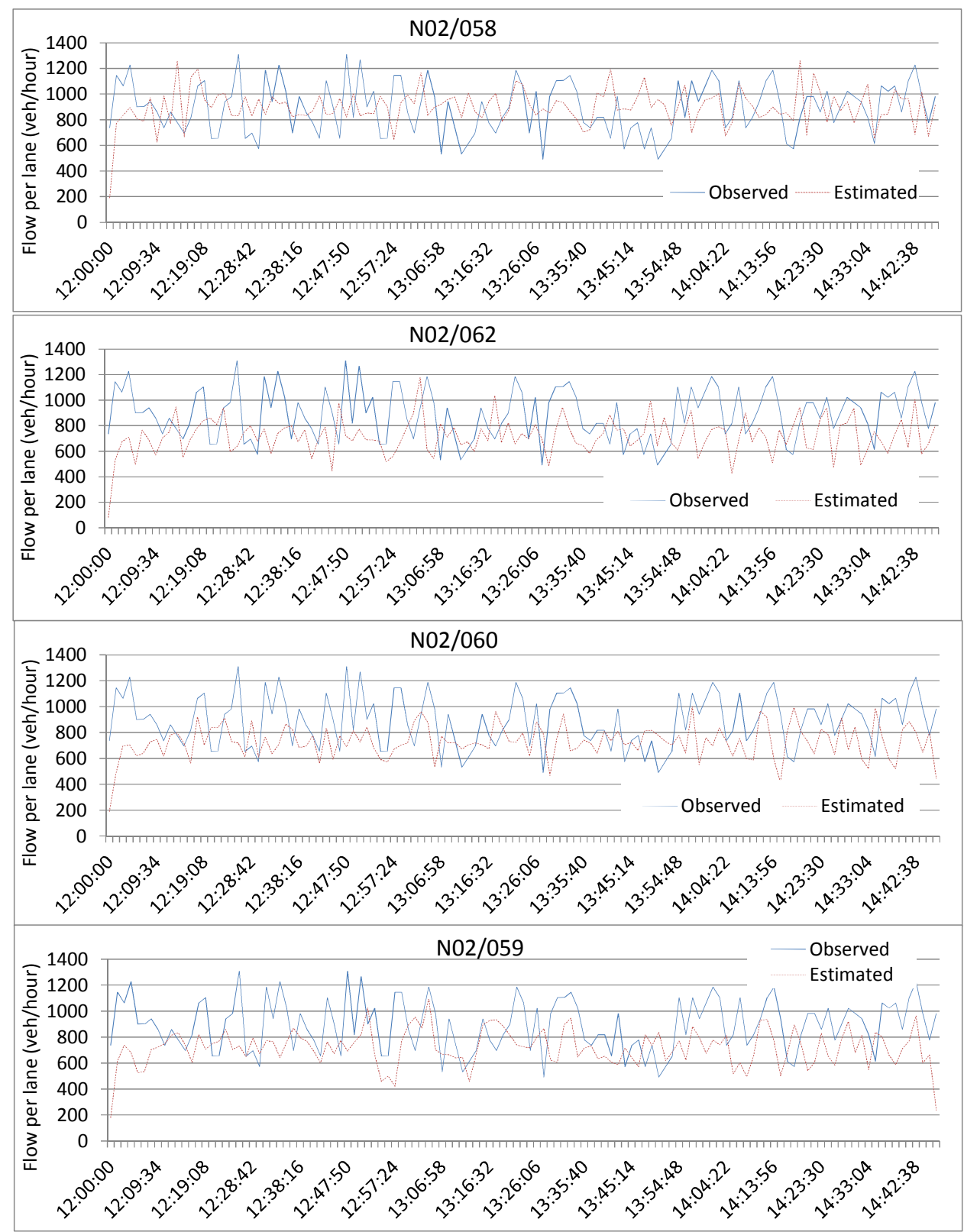

Figure 13: Flow estimates by CTM with three-segment fundamentals 


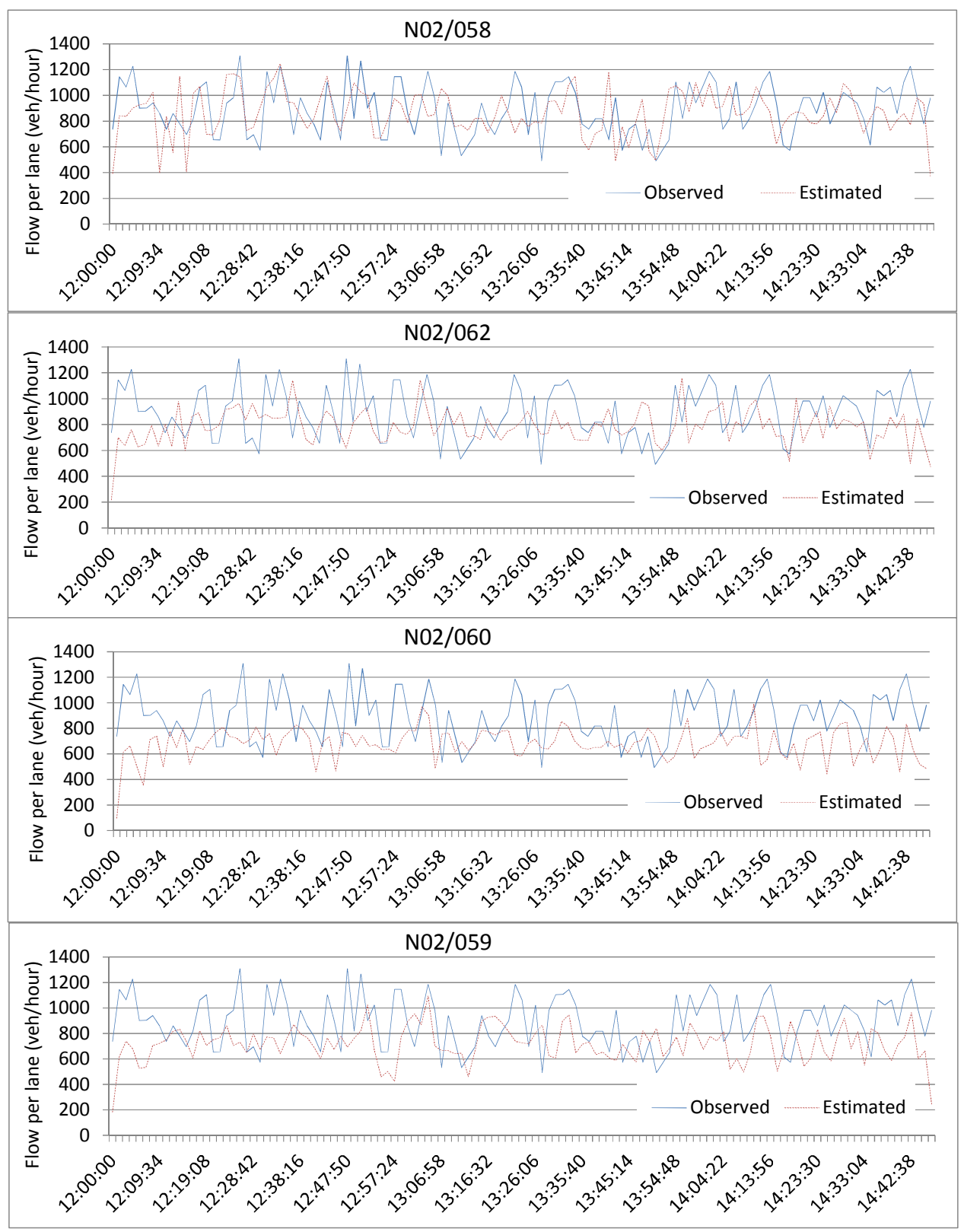

Figure 14: Flow estimates by variational method with three-segment fundamentals 